\title{
Low-Overhead Feedback Topology Design for the K-User MIMO Interference Alignment
}

\author{
Jin $\mathrm{Jin}^{1^{*}}$, Xiang-Chuan $\mathrm{Gao}^{1}$, Xingwang $\mathrm{Li}^{2}$, Charles C. Cavalcante ${ }^{3}$ and Lihua $\mathrm{Li}^{4}$ \\ ${ }^{1}$ School of Information Engineering, Zhengzhou University \\ Zhengzhou 450001, China \\ [e-mail: iejjin@zzu.edu.cn, iexcgao@zzu.edu.cn] \\ ${ }^{2}$ School of Physics and Electronic Information Engineering, Henan Polytechnic University \\ Jiaozuo 454000, China \\ [e-mail: lixingwangbupt@gmail.com] \\ ${ }^{3}$ Wireless Telecommunications Research Group, Federal University of Ceará \\ Campus do Pici, Bl. 722, ZIP 60455-760, Fortaleza-CE, Brazil \\ [e-mail: charles@gtel.ufc.br] \\ ${ }^{4}$ State Key Laboratory of Networking and Switching Technology, Beijing University of Posts and \\ Telecommunications, Beijing 100876, China \\ [e-mail: lilihua@bupt.edu.cn] \\ *Corresponding author: Jin Jin
}

Received November 2, 2017; revised May 22, 2018; accepted June 22, 2018;

published November 30, 2018

\begin{abstract}
Since designing a feedback topology is a practical way to implement interference alignment at reduced cost of channel state information (CSI) feedback, six feedback topologies have been presented in prior works for a K-user multiple-input multiple-output interference channel. To fully reveal the potential benefits of the feedback topology in terms of the saving of CSI overhead, we propose a new feedback topology in this paper. By efficiently performing dimensionality-decreasing at the transmitter side and aligning interference signals at a subset of receivers, we show that the proposed feedback topology obtains substantial reduction of feedback cost over the existing six feedback designs under the same antenna configuration.
\end{abstract}

Keywords: Interference alignment, channel state information, feedback topology, interference channel, multiple-input-multiple-output

This work was supported in part by the National Natural Science Foundation of China under Grant 61501404, and Grant 61640003; in part by the Major Projects in Henan Province under Grant 161100210200; in part by the Henan Scientific and Technological Research Project under Grant 182102210307; in part by the Outstanding Young Talent Research Fund of ZZU under Grant 1521318003; in part by the CNPq-Brazil (Procs. 309472/2017-2 and 408609/2016-8). 


\section{Introduction}

Interference alignment (IA) is an advanced technique for multi-node coordination as it is optimal in terms of degrees of freedom (DoF) at high signal-to-noise ratio regime, and thus IA can be exploited in interference channel [1], relay based wireless networks [2-3], heterogeneous networks [4], etc. In fact, the DoF gains of IA are obtained by designing IA precoders whose computations require sufficient channel state information (CSI) at the transmitters (Txs). To determine IA precoders, two types of approaches can be applied to tackle the CSI acquisition issue: reciprocity and feedback [5]. For time division duplex (TDD) systems where ideal uplink-downlink reciprocity of the radio channels is assumed, after each base station acquires the channel knowledge for its downlink channels by estimating the uplink counterparts, these local CSIs are exchanged between the base stations so as to calculate IA precoders [6-7]. However, in practice, channel reciprocity cannot be directly utilized due to radio frequency circuits discrepancy, so that a TDD system needs to be tightly calibrated before exploiting reciprocity [8-9]. On the other hand, for frequency division duplex (FDD) systems where reciprocity does not hold, each receiver (Rx) feeds back the information about the estimated CSIs to all Txs for the implementation of IA [10-11]. This feedback mechanism is called the full-feedback topology in [12], which incurs an unacceptable feedback overhead penalty.

For the K-user multiple-input-multiple-output (MIMO) interference channel, due to the impracticality of the excessively high-overhead feedback in the full-feedback topology, two centralized feedback topologies and a CSI-exchange topology were provided in [12] to reduce the CSI feedback cost to a reasonable level. Then in [13], three improved feedback structures were presented to lower the CSI overhead or decrease the time delay for those feedback designs in [12]. Consider the existing six feedback topologies. The two-hop centralized topologies either impose heavy computation and feedback burden on a CSI collecting Rx or introduce an additional CSI control station. By separating the centralized task of computation and feedback for all precoders at one designated node into a few distributed subtasks at several designated Rxs, the distributed feedback topology not only overcomes the above drawbacks of the centralized structures, but also attains dramatic reduction of CSI overhead. In addition, by simply re-arranging the information exchange order, the modified CSI-exchange design shortens transmission delay in the original CSI-exchange design. Besides, the four-hop feedback topology, which employs 4 time slots to determine all IA precoders, has the lowest CSI overhead among the existing six feedback structures.

From [14, Table V], designing a feedback topology is one of the effective methods for solving the CSI acquisition problem. Although six feedback structures have already been provided based on specific closed-form IA solutions in the field of feedback topology construction, it does not mean that the potential advantage of feedback topologies in the saving of CSI overhead has been completely revealed. In other words, it is possible to design a new feedback structure with less CSI overhead by finding an appropriate closed-form IA solution different from those used in the existing six feedback topologies, because multiple IA solutions exist in the K-user MIMO interference channel [15-16]. For this reason, in this paper, we concentrate on the CSI overhead reduction from the perspective of feedback topology design based on closed-form IA solution.

Note that a number of research works are devoted to the reduction of feedback overhead for IA [17-24], among which, two related works focusing on the MIMO interference channel have 
been done in [17] and [18]. One difference between the feedback topology in this work and the schemes in [17] and [18] is that the proposed feedback topology allows CSI exchange between the Rxs (this Rx cooperation is also exploited in [12] and [13]), while the case of no Rx cooperation is investigated in [17] and [18]. Additionally, the IA precoders in [17] and [18] are computed by iterative approaches under arbitrary antenna configurations, whereas the precoders in the feedback topologies studied in [12-13] and this work, are derived by closed-form IA solutions under specific antenna configurations. Compared to the closed-form solutions, the iterative IA approaches have significantly high computational complexity. Therefore, the closed-form IA solutions are more suitable for practical implementation than the iterative algorithms. This motivates us to pursue the saving of CSI overhead in the field of feedback topology design.

In this paper, we consider the MIMO interference channel under the same antenna configuration as that discussed in [12] and [13]. The main contributions of this paper are summarized as follows: 1) We develop a specific closed-form IA solution with which a new feedback structure is proposed. By making partial interference signals aligned at a subset of Rxs after efficiently exploiting dimensionality-decreasing at the Tx side, the proposed feedback structure requires lower CSI overhead to complete the computation and delivery of IA precoders compared to the existing feedback designs. 2) Since feedback topology is classified as one of the methods aiming at overcoming the CSI problem of IA [14], we further reveal the advantage of feedback topology in terms of CSI overhead reduction by providing the proposed low-overhead feedback topology.

The remainder of this paper is organized as follows. The system model is introduced in Section 2. In Section 3, we present the proposed feedback topology. Section 4 compares the proposed feedback topology to the existing designs. Conclusions are given in Section 5.

\section{System Model}

The K-user MIMO interference channel is comprised of $K \mathrm{Tx}$-Rx pairs with $M$ antennas at each node. Each Tx exploits a linear precoder to transmit $d$ data streams to its corresponding $\mathrm{Rx}$. Here, the antenna configuration of $M=(K-1) d$ for $K \geq 4$ is considered in this paper as in [13]. Denoting $\mathbf{y}_{i}$ as the signal received at $\mathrm{Rx} i, \mathbf{y}_{i}$ can be written as

$$
\mathbf{y}_{i}=\sqrt{\frac{P}{d}} \mathbf{H}_{i i} \mathbf{V}_{i} \mathbf{s}_{i}+\sqrt{\frac{P}{d}} \sum_{j=1, j \neq i}^{K} \mathbf{H}_{i j} \mathbf{V}_{j} \mathbf{s}_{j}+\mathbf{n}_{i}
$$

where $\mathbf{n}_{i}$ denotes the additive white Gaussian noise vector with the covariance matrix $\mathbf{I}_{M}$ observed at $\operatorname{Rx} i, \mathbf{s}_{i}=\left[\mathbf{s}_{i}^{[1]} \cdots \mathbf{s}_{i}^{[d]}\right]^{T}$ stands for the $d \times 1$ data vector with each entry having zero mean and unit variance transmitted from $\mathrm{Tx} i, \mathbf{V}_{i}=\left[\mathbf{v}_{i}^{[1]} \cdots \mathbf{v}_{i}^{[d]}\right]$ is the precoder of size $M \times d$ at Tx $i, P$ denotes the transmission power, and $\mathbf{H}_{i j}$ indicates the channel matrix of size $M \times M$ from Tx $j$ to $\mathrm{Rx} i$. It is assumed that the channel elements are sampled from independent identically distributed (i.i.d.) complex Gaussian random variables with zero mean and unit variance. In addition, the receive filter of size $M \times d$ at $\mathrm{Rx} i$ is denoted as $\mathbf{U}_{i}=\left[\mathbf{u}_{i}^{[1]} \cdots \mathbf{u}_{i}^{[d]}\right]$. As in [12], we assume that each column of the precoder $\mathbf{V}_{i}$ and each column of the receive filter $\mathbf{U}_{i}$ are normalized to have unit norm, i.e., $\left\|\mathbf{v}_{i}^{[l]}\right\|^{2}=\left\|\mathbf{u}_{i}^{[l]}\right\|^{2}=1$ for 
$\forall l \in\{1, \cdots, d\}$. Through the proposed transceiver design, all interference signals can be cancelled completely, so that the achievable sum rate is given by

$$
R_{\text {sum_rate }}=\sum_{i=1}^{K} \sum_{l=1}^{d} \log _{2}\left(1+\frac{P}{d}\left|\left(\mathbf{u}_{i}^{[l]}\right)^{H} \mathbf{H}_{i i} \mathbf{v}_{i}^{[l]}\right|^{2}\right) .
$$

Notably, in this paper, the FDD system is taken into account, and the CSI overhead refers to the total number of complex coefficients used for the exchange of CSI and precoders as in [12] and [13].

\section{Low-Overhead Feedback Topology}

In this section, we first briefly describe the key idea of the proposed feedback design. Then, we introduce the proposed low-overhead feedback topology for the K-user MIMO interference channel under the antenna configuration of $M=(K-1) d$.

\subsection{The Key to Proposed Feedback Topology}

From [18], the antenna configuration of $M=(K-1) d$ considered here is a super-feasible antenna setting where each node has redundant antennas which can be ignored without affecting the DoF characterization. Therefore, if we ignore some of these redundant antennas, it is possible to reduce the overhead of CSI feedback because the number of complex coefficients of the channel matrix becomes small. Two methods can be used for ignoring $M-N$ (where $d \leq N<M$ ) antennas at a Tx: randomly selecting $N$ antennas while powering off others, and using a randomly picked auxiliary precoder of size $M \times N$. For the sake of clarity in description of the IA precoder design for the feedback topology, here we choose the precoding method. After Tx $j$ sends a randomly picked auxiliary precoder $\mathbf{v}_{j} \in \mathrm{C}^{M \times N}$, the effective channel matrix $\mathbf{H}_{i j} \mathbf{v}_{j}$ seen at $\mathrm{Rx} i, \forall i$, is of size $M \times N$. This implies that we can equivalently think that the spatial dimensions provided by the transmit antennas at Tx $j$ using a randomly picked precoder is decreased from $M$ to $N$. We refer to this precoding process as dimensionality-decreasing at the Tx side. It is worth noting that a precoder of size $M \times d$ can be formulated as two cascaded precoders in the proposed feedback design. The preceding precoder named as auxiliary precoder is used to perform dimensionality-decreasing, eliminate interference, or zero force interference after implementing dimensionality-decreasing, while the subsequent precoder named as combining precoder is designed to align interference.

Now, let us roughly review the precoder designs of the existing six feedback structures. The four-hop topology carries out dimensionality-decreasing by randomly picking the precoders of $(K-3)$ designated Txs, and constructs the precoders of other three Txs so as to let two interference signals be aligned at each of the three designated Rxs, whereas the other existing feedback topologies design all precoders to ensure that two cross-link interferences are overlapped at each of the $K$ Rxs. Due to the fact that the CSI overhead of the four-hop topology is lower than those of other existing feedback designs, the four-hop topology is able to benefit from its distinctive precoder design to achieve a CSI overhead reduction.

Inspired by the four-hop feedback topology, in this work we aim to establish a new feedback topology to seek a further saving of CSI overhead. The key idea behind the proposed feedback topology is an efficient way to exploit the dimensionality-decreasing at the Tx side, and align 
interference signals at a subset of Rxs. To be specific, for the proposed feedback topology, we employ the dimensionality-decreasing in a flexible fashion to allow a certain Tx to remain $N$ (where $d \leq N<M$ ) dimensions after ignoring $M-N$ redundant antennas. Additionally, in order to let a subset of Rxs observe aligned interference signals, the precoders used for implementing alignment in the proposed topology can be computed in different time slots. In contrast, for the four-hop feedback structure, the number of remaining dimensions at a certain Tx after applying the dimensionality-decreasing is fixed to $d$, and the precoders used for carrying out alignment are calculated during one time slot.

Relying on the proposed idea, we will provide the detailed design of the new feedback topology in the following subsections. The proposed feedback topology consists of the derivation of the closed-form IA solution, and the exchange of information used for acquiring precoders at the Tx side. Our topology is carried out in three phases. In phase 1, using two time slots, the precoders of size $M \times d$ for Txs $1,2, \ldots,(K-2)$ are obtained at the corresponding TXs, and then the cross-link interferences from these $(K-2)$ Txs are eliminated at the Rx side. In phase 2, employing one time slot, $\mathrm{Tx}(K-1)$ and $\mathrm{Tx} K$ acquire their corresponding auxiliary precoders of size $M \times 2 d$. In phase 3 , by spending three time slots, the combining precoders of size $2 d \times d$ are attained at Tx $(K-1)$ and Tx $K$. To facilitate the exposition, we start with the $K=4$ case.

\subsection{Proposed Feedback Topology for $K=4$}

In this subsection, we consider the case of $K=4$ to explain the key idea of the proposed feedback topology in detail. For this case, there are 12 cross-links in the system, so that the precoders and receive filters are designed to cancel all cross-link interferences as illustrated in Fig. 1. Note that, in Fig. 1 (also in Fig. 3 and Fig. 5), the directions of green arrows indicate the nodes that implement zero-forcing to nullify interference signals, and the red arrows indicate the aligned effective interference channels.

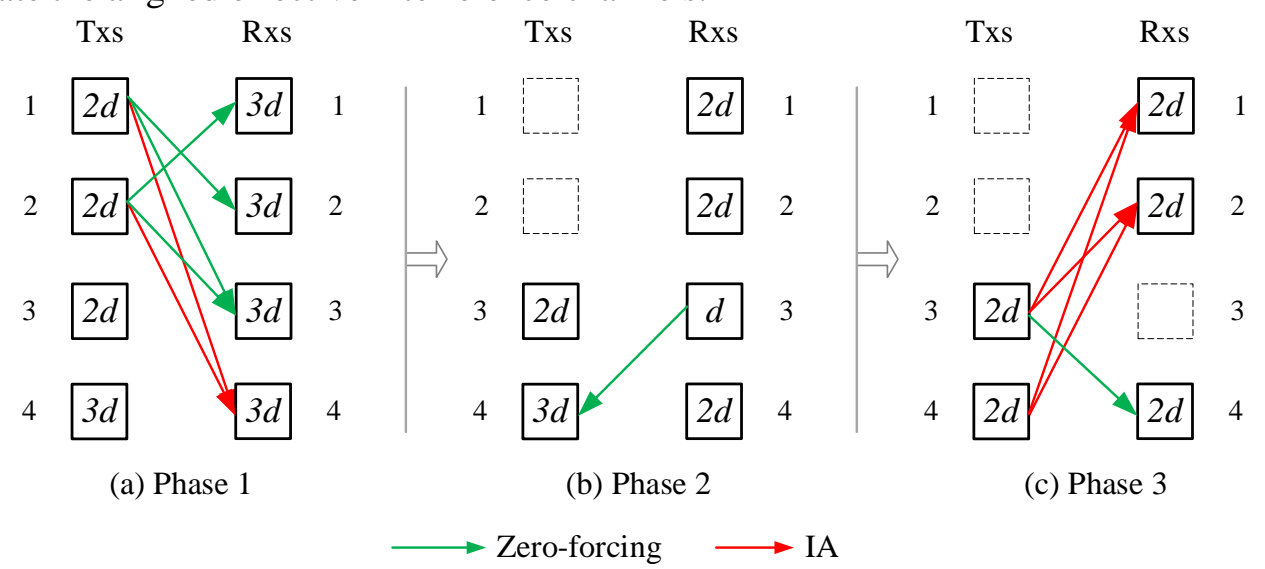

Fig. 1. Illustration of how to eliminate all cross-link interferences when $K=4$

For the case of $K=4$, each node is equipped with $3 d$ antennas. In order to implement the dimensionality-decreasing at the Tx side, we randomly generate the auxiliary precoders $\mathbf{v}_{1} \in \mathrm{C}^{3 d \times 2 d}, \mathbf{v}_{2} \in \mathrm{C}^{3 d \times 2 d}$, and $\mathbf{v}_{3} \in \mathrm{C}^{3 d \times 2 d}$ for Tx 1, Tx 2, and Tx 3, respectively. After applying these auxiliary precoders, we can equivalently think that the numbers of antennas at Txs 1,2 , and 3 become $2 d$. Then Txs 1 and 2 send precoded pilot symbols using their 
predetermined auxiliary precoders $\mathbf{v}_{1}$ and $\mathbf{v}_{2}$, respectively. Remarkably, the initial pilot sending procedure is not involved in the total required time slots for the feedback structures in [12] and [13]. Accordingly, when counting the number of time slots needed for the proposed feedback design, we do not consider the time slot used for the initial pilot transmission either. Therefore, the proposed topology for $K=4$ demands 6 time slots in total to complete the calculation and delivery of IA precoders. The detailed design process is explained as follows.

1) Phase 1: Two time slots are employed in this phase. In time slot 1, after Rx 4 estimates the interfering effective channels from Txs 1 and 2 (i.e., $\mathbf{H}_{41} \mathbf{v}_{1}$ and $\mathbf{H}_{42} \mathbf{v}_{2}$ ), as depicted in Fig. 1(a), two combining precoders $\tilde{\mathbf{v}}_{1} \in \mathrm{C}^{2 d \times d}$ and $\tilde{\mathbf{v}}_{2} \in \mathrm{C}^{2 d \times d}$ are designed to make the interfering links from Txs 1 and 2 to Rx 4 span the same subspace:

$$
\operatorname{span}\left(\mathbf{H}_{41} \mathbf{v}_{1} \tilde{\mathbf{v}}_{1}\right)=\operatorname{span}\left(\mathbf{H}_{42} \mathbf{v}_{2} \tilde{\mathbf{v}}_{2}\right)
$$

where $\operatorname{span}(\cdot)$ denotes the space spanned by the column vectors of a matrix. To satisfy this condition, we formulate the following matrix equation,

$$
\left[\begin{array}{ll}
\mathbf{H}_{41} \mathbf{v}_{1}-\mathbf{H}_{42} \mathbf{v}_{2}
\end{array}\right]\left[\begin{array}{c}
\tilde{\mathbf{v}}_{1} \\
\tilde{\mathbf{v}}_{2}
\end{array}\right]=\mathbf{F G}=0 .
$$

Since the size of the matrix $\mathbf{F}$ is $3 d \times 4 d$, $\mathbf{G}$ can be chosen as $d$ basis vectors of the null space of $\mathbf{F}$, from which $\tilde{\mathbf{v}}_{1}$ and $\tilde{\mathbf{v}}_{2}$ can be acquired at $\mathrm{Rx} 4$. Then the two computed combining precoders are fed back from $\mathrm{Rx} 4$ to the corresponding Txs, and thus we have $\mathbf{V}_{1}=\mathbf{v}_{1} \tilde{\mathbf{v}}_{1}$ and $\mathbf{V}_{2}=\mathbf{v}_{2} \tilde{\mathbf{v}}_{2}$ at Txs 1 and 2, respectively.

In the second time slot, as illustrated in Fig. 2, Txs 1 and 2 forward precoded pilot symbols using the obtained precoders $\mathbf{V}_{1} \in \mathrm{C}^{3 d \times d}$ and $\mathbf{V}_{2} \in \mathrm{C}^{3 d \times d}$ respectively, while Tx 4 transmits non-precoded pilots at the same time. Hence, Rx $i, i \in\{1,2,3,4\}$, is capable of estimating the interfering effective channels $\mathbf{H}_{i j} \mathbf{V}_{j}(j=1,2$ and $j \neq i)$. Now, we design the auxiliary receive filters $\mathbf{u}_{1} \in \mathrm{C}^{3 d \times 2 d}, \mathbf{u}_{2} \in \mathrm{C}^{3 d \times 2 d}, \mathbf{u}_{4} \in \mathrm{C}^{3 d \times 2 d}$ for Rxs 1 , 2, 4, respectively, and the receive filter $\mathbf{U}_{3} \in \mathrm{C}^{3 d \times d}$ for $\mathrm{Rx} 3$, so as to suppress the interferences caused by Tx 1 and Tx 2 at the Rx side.

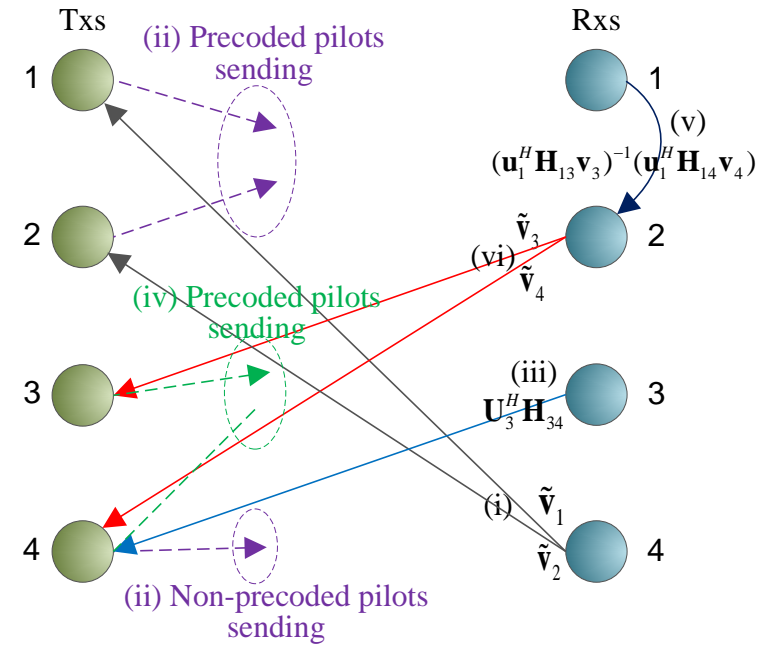

Fig. 2. Illustration of the CSI signaling exchange procedure for the proposed feedback topology when

$$
K=4
$$


For $\mathrm{Rx} 1$, the auxiliary receive filter $\mathbf{u}_{1}$ can be calculated as

$$
\mathbf{u}_{1} \subset \operatorname{null}\left(\mathbf{V}_{2}^{H} \mathbf{H}_{12}^{H}\right)
$$

where $\operatorname{null}(\cdot)$ denotes an orthonormal basis for the null space of a matrix. Since $\mathbf{V}_{2}^{H} \mathbf{H}_{12}^{H}$ is a $d \times 3 d$ matrix, $\mathbf{u}_{1}$ is chosen as $2 d$ basis vectors of the null space of $\mathbf{V}_{2}^{H} \mathbf{H}_{12}^{H}$.

In a similar fashion, the auxiliary receive filter $\mathbf{u}_{2}$ for $\mathrm{Rx} 2$ can be obtained as

$$
\mathbf{u}_{2} \subset \operatorname{null}\left(\mathbf{V}_{1}^{H} \mathbf{H}_{21}^{H}\right)
$$

where $\mathbf{V}_{1}^{H} \mathbf{H}_{21}^{H}$ is a $d \times 3 d$ matrix which has a $2 d$-dimensional null space. Thus, $\mathbf{u}_{2}$ is determined as $2 d$ basis vectors of the null space of $\mathbf{V}_{1}^{H} \mathbf{H}_{21}^{H}$.

Next, the receive filter $\mathbf{U}_{3}$ for $\mathrm{Rx} 3$ is given by

$$
\mathbf{U}_{3} \subset \operatorname{null}\left(\left[\left(\mathbf{V}_{1}^{H} \mathbf{H}_{31}^{H}\right)^{H}\left(\mathbf{V}_{2}^{H} \mathbf{H}_{32}^{H}\right)^{H}\right]^{H}\right) .
$$

Here, $\mathbf{U}_{3}$ can be obtained as $d$ basis vectors of the null space of $\left[\left(\mathbf{V}_{1}^{H} \mathbf{H}_{31}^{H}\right)^{H}\left(\mathbf{V}_{2}^{H} \mathbf{H}_{32}^{H}\right)^{H}\right]^{H}$.

Because the interfering links from Txs 1 and 2 to $\mathrm{Rx} 4$ are aligned with each other, $\mathrm{Rx} 4$ is able to consider two different interferers as one interferer which spans $d$ dimensional subspace. Therefore, the auxiliary receive filter $\mathbf{u}_{4}$ can be constructed as $2 d$ linearly independent vectors of the null space of $\mathbf{V}_{1}^{H} \mathbf{H}_{41}^{H}$ :

$$
\mathbf{u}_{4} \subset \operatorname{null}\left(\mathbf{V}_{1}^{H} \mathbf{H}_{41}^{H}\right) \text {. }
$$

So far, 6 interfering links from Tx 1 and Tx 2 are zero forced at the unintended Rxs. Notably, once all interfering links connected to a certain node have been eliminated, this node is discarded from the system in Fig. 1 (also in Fig. 3 and Fig. 5) for simplicity of exposition.

2) Phase 2: One time slot is used in phase 2. Recall that Tx 4 conveys non-precoded pilots during the second time slot. This means that $\mathrm{Rx} 3$ is capable of estimating the cross-link channel from Tx 4 to Rx 3, i.e., $\mathbf{H}_{34}$. In time slot 3, Rx 3 feeds back $\mathbf{U}_{3}^{H} \mathbf{H}_{34}$ of size $d \times 3 d$ to Tx 4. As shown in Fig. 1(b), the design goal of the auxiliary precoders $\mathbf{v}_{4} \in \mathrm{C}^{3 d \times 2 d}$ is to cancel the interfering link between $\mathrm{Tx} 4$ and $\mathrm{Rx}$ 3, i.e.,

$$
\mathbf{v}_{4} \subset \operatorname{null}\left(\mathbf{U}_{3}^{H} \mathbf{H}_{34}\right) \text {. }
$$

Simply, $\mathbf{v}_{4}$ is determined as $2 d$ basis vectors of the null space of $\mathbf{U}_{3}^{H} \mathbf{H}_{34}$, and thus Tx 4 obtains its auxiliary precoder $\mathbf{v}_{4}$. Besides, note that the randomly generated auxiliary precoder $\mathbf{v}_{3}$ is already acquired at Tx 3 through the dimensionality-decreasing process.

3) Phase 3: This phase spans 3 time slots. In time slot 4 , Txs 3 and 4 transmit precoded pilot symbols using the obtained auxiliary precoders $\mathbf{v}_{3}$ and $\mathbf{v}_{4}$ respectively, so that Rx 1 and Rx 2 can estimate the interfering channels from Txs 3 and 4 . Now, as illustrated in Fig. 1(c), at each of Rx 1 and $\mathrm{Rx} 2$, the interferences caused by Txs 3 and 4 should be aligned in the same subspace. To this end, two combining precoders $\tilde{\mathbf{v}}_{3} \in \mathrm{C}^{2 d \times d}$ and $\tilde{\mathbf{v}}_{4} \in \mathrm{C}^{2 d \times d}$ are designed to 
satisfy the following interference aligning conditions:

$$
\begin{aligned}
& \operatorname{span}\left(\mathbf{u}_{1}^{H} \mathbf{H}_{13} \mathbf{v}_{3} \tilde{\mathbf{v}}_{3}\right)=\operatorname{span}\left(\mathbf{u}_{1}^{H} \mathbf{H}_{14} \mathbf{v}_{4} \tilde{\mathbf{v}}_{4}\right), \\
& \operatorname{span}\left(\mathbf{u}_{2}^{H} \mathbf{H}_{23} \mathbf{v}_{3} \tilde{\mathbf{v}}_{3}\right)=\operatorname{span}\left(\mathbf{u}_{2}^{H} \mathbf{H}_{24} \mathbf{v}_{4} \tilde{\mathbf{v}}_{4}\right) .
\end{aligned}
$$

The combining precoders in (10) can be computed as

$$
\begin{aligned}
& \tilde{\mathbf{v}}_{3}=\operatorname{eig}\left(\left(\mathbf{u}_{1}^{H} \mathbf{H}_{13} \mathbf{v}_{3}\right)^{-1}\left(\mathbf{u}_{1}^{H} \mathbf{H}_{14} \mathbf{v}_{4}\right)\left(\mathbf{u}_{2}^{H} \mathbf{H}_{24} \mathbf{v}_{4}\right)^{-1}\left(\mathbf{u}_{2}^{H} \mathbf{H}_{23} \mathbf{v}_{3}\right)\right), \\
& \tilde{\mathbf{v}}_{4}=\left(\mathbf{u}_{2}^{H} \mathbf{H}_{24} \mathbf{v}_{4}\right)^{-1}\left(\mathbf{u}_{2}^{H} \mathbf{H}_{23} \mathbf{v}_{3}\right) \tilde{\mathbf{v}}_{3},
\end{aligned}
$$

where $\operatorname{eig}(\mathbf{A})$ stands for the matrix containing $d$ eigenvectors of $\mathbf{A}$.

In time slot 5, the matrix $\left(\mathbf{u}_{1}^{H} \mathbf{H}_{13} \mathbf{v}_{3}\right)^{-1}\left(\mathbf{u}_{1}^{H} \mathbf{H}_{14} \mathbf{v}_{4}\right)$ of size $2 d \times 2 d$ is delivered from Rx 1 to Rx 2, and thus Rx 2 is able to obtain $\tilde{\mathbf{v}}_{3}$ and $\tilde{\mathbf{v}}_{4}$ according to (11).

In time slot 6, Rx 2 feeds back $\tilde{\mathbf{v}}_{3}$ and $\tilde{\mathbf{v}}_{4}$ to the corresponding Txs, so that $\mathbf{V}_{3}=\mathbf{v}_{3} \tilde{\mathbf{v}}_{3}$ is acquired at Tx 3 and $\mathbf{V}_{4}=\mathbf{v}_{4} \tilde{\mathbf{v}}_{4}$ is obtained at Tx 4. Now, let us consider the remaining interfering links which are not cancelled so far. As can be seen in Fig. 1(c), the interference signals from Txs 3 and 4 are aligned together at each of Rxs 1 and 2, so that each of Rx 1 and $\mathrm{Rx} 2$ with $2 d$ spatial dimensions created by the $2 d$ remaining antennas can keep its desired signal separate from the aligned interferences. Besides, $\mathrm{Rx} 4$ with $2 d$ remaining antennas is able to guarantee $d$ interference-free dimensions after using zero-forcing to discard $d$ dimensions occupied by the interference from Tx 3 to $\mathrm{Rx} 4$.

Table 1. Implementation of the proposed feedback topology for $K=4$

(a) The combining precoders $\tilde{\mathbf{v}}_{1}$ and $\tilde{\mathbf{v}}_{2}$ of size $2 d \times d$ are sent from $\mathrm{Rx} 4$ to the corresponding Txs;

(b) Txs 1, 2 and 4 transmit pilot symbols;

(c) The $d \times 3 d$ matrix $\mathbf{U}_{3}^{H} \mathbf{H}_{34}$ is delivered from $\operatorname{Rx} 3$ to Tx 4;

(d) Tx 3 and Tx 4 forward pilot symbols;

(e) Rx 1 conveys $\left(\mathbf{u}_{1}^{H} \mathbf{H}_{13} \mathbf{v}_{3}\right)^{-1}\left(\mathbf{u}_{1}^{H} \mathbf{H}_{14} \mathbf{v}_{4}\right)$ of size $2 d \times 2 d$ to Rx 2;

(f) Rx 2 feeds back $\tilde{\mathbf{v}}_{3}$ and $\tilde{\mathbf{v}}_{4}$ of size $2 d \times d$ to the corresponding Txs.

Finally, it is clear that a total of 12 cross-link interferences can be zero forced completely via the transceiver design shown in Fig. 1. Remarkably, in [12] and [13], counting the required number of time slots for the feedback topologies is terminated once all IA precoders are obtained at the Tx side. Obeying this counting criterion, 6 time slots are needed for the proposed feedback structure for $K=4$ to implement IA, as shown in Table 1. In summary, adding up the CSI overhead in (a), (c), (e) and (f) in Table 1, the total CSI overhead in the proposed feedback topology for the case of $K=4$ is $15 d^{2}$.

\subsection{Proposed Feedback Topology for $K=5$}

For the $K=5$ case where each node has $4 d$ antennas, dimensionality-decreasing is 
performed at each of the $K$ Txs. It is worth pointing out that, in this case each of the auxiliary precoders of Tx 4 and Tx 5 is given as two cascaded precoders: the first auxiliary precoder is employed to carry out dimensionality-decreasing, and the second auxiliary precoder is designed to zero force interference. Here, we randomly pick the precoder $\mathbf{V}_{1} \in C^{4 d \times d}$ for $T x 1$, the auxiliary precoders $\mathbf{v}_{2} \in \mathrm{C}^{4 d \times 2 d}, \mathbf{v}_{3} \in \mathrm{C}^{4 d \times 3 d}$ for Tx 2 and $\mathrm{Tx} 3$ respectively, and the first auxiliary precoders $\mathbf{v}_{4}^{(1)} \in \mathrm{C}^{4 d \times 3 d}, \mathbf{v}_{5}^{(1)} \in \mathrm{C}^{4 d \times 3 d}$ for Tx 4 and Tx 5 respectively.

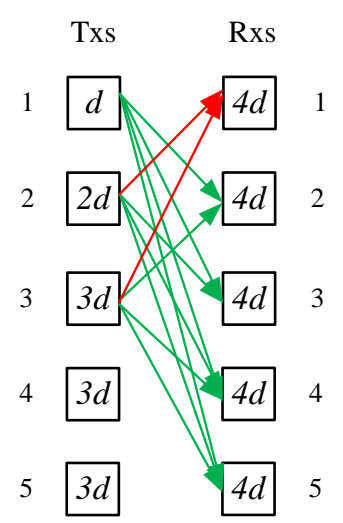

(a) Phase 1

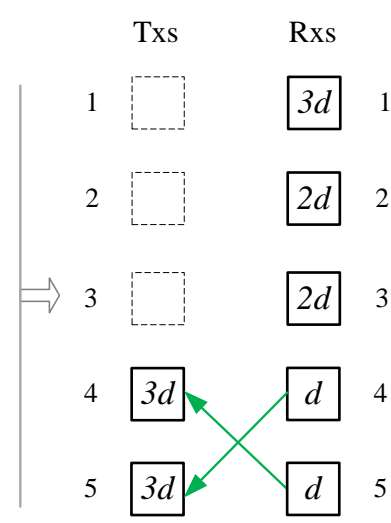

(b) Phase 2

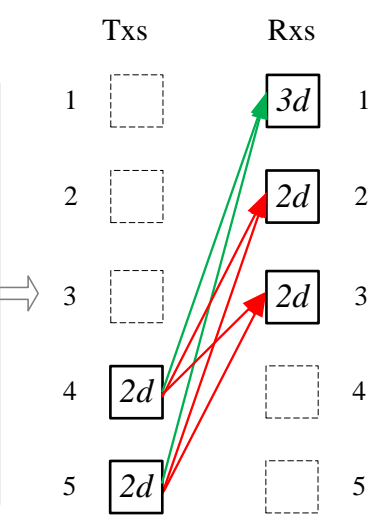

(c) Phase 3

Zero-forcing IA

Fig. 3. Illustration of how to eliminate all cross-link interferences when $K=5$

1) Phase 1: After applying the auxiliary precoders $\mathbf{v}_{2}$ and $\mathbf{v}_{3}$ at Txs 2 and 3 respectively, as depicted in Fig. 3(a), the interfering links from Txs 2 and 3 to $\mathrm{Rx} 1$ can be aligned together via the design of the two combining precoders $\tilde{\mathbf{v}}_{2} \in C^{2 d \times d}$ and $\tilde{\mathbf{v}}_{3} \in \mathrm{C}^{3 d \times d}$ which satisfy the condition:

$$
\left[\begin{array}{lll}
\mathbf{H}_{12} \mathbf{v}_{2} & -\mathbf{H}_{13} \mathbf{v}_{3}
\end{array}\right]\left[\begin{array}{c}
\tilde{\mathbf{v}}_{2} \\
\tilde{\mathbf{v}}_{3}
\end{array}\right]=0 .
$$

Since the size of the matrix $\left[\mathbf{H}_{12} \mathbf{v}_{2}-\mathbf{H}_{13} \mathbf{v}_{3}\right]$ is $4 d \times 5 d$, it turns out that $\tilde{\mathbf{v}}_{2}$ and $\tilde{\mathbf{v}}_{3}$ can be computed definitely. Once the two combining precoders $\tilde{\mathbf{v}}_{2}$ and $\tilde{\mathbf{v}}_{3}$ are delivered from $\mathrm{Rx} 1$ to the corresponding Txs, the two precoders $\mathbf{V}_{2}=\mathbf{V}_{2} \tilde{\mathbf{v}}_{2}$ and $\mathbf{V}_{3}=\mathbf{V}_{3} \tilde{\mathbf{v}}_{3}$ are constructed at Tx 2 and Tx 3, respectively. 


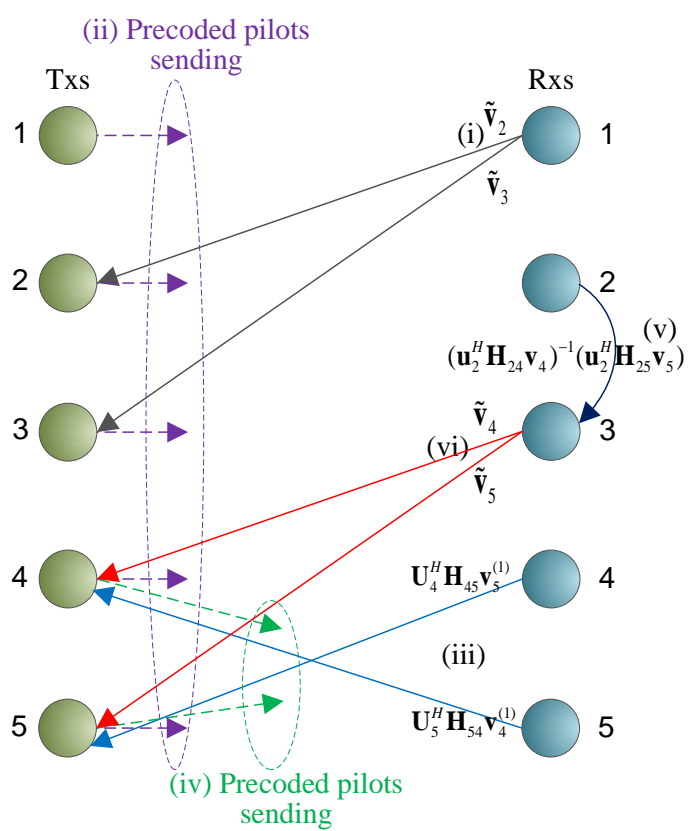

Fig. 4. Illustration of the CSI signaling exchange procedure for the proposed feedback topology when $K=5$

Then, as shown in Fig. 4, each of Txs 1, 2 and 3 transmits precoded pilot symbols using its precoder of size $4 d \times d$, while Tx 4 and Tx 5 send precoded pilot symbols using $\mathbf{v}_{4}^{(1)}$ and $\mathbf{v}_{5}^{(1)}$ respectively. After channel estimation, all interferences caused by Txs 1, 2 and 3 can be eliminated at the Rx side as shown in Fig. 3(a). To this end, the receive filter (or auxiliary receive filter) of $\mathrm{Rx} n, n \in\{1,2,3,4,5\}$, is designed to lie in the null space of the interference subspace containing the cross-link interferences from Txs 1, 2 and 3 to $\mathrm{Rx} n$. In this way, the auxiliary receive filters $\mathbf{u}_{1} \in C^{4 d \times 3 d}, \mathbf{u}_{2} \in C^{4 d \times 2 d}, \mathbf{u}_{3} \in C^{4 d \times 2 d}$, and the receive filters $\mathbf{U}_{4} \in \mathrm{C}^{4 d \times d}, \mathbf{U}_{5} \in \mathrm{C}^{4 d \times d}$, are determined at the corresponding Rxs. Through the design of these receive filters (or auxiliary receive filters), we can equivalently think that Rxs $1,2,3,4$ and 5 discard $d, 2 d, 2 d, 3 d$ and $3 d$ dimensions respectively.

2) Phase 2: During this phase, after $\operatorname{Rx} 4$ estimates the effective channel between $T x 5$ and Rx 4, i.e., $\mathbf{H}_{45} \mathbf{v}_{5}^{(1)}$, it feeds back $\mathbf{U}_{4}^{H} \mathbf{H}_{45} \mathbf{v}_{5}^{(1)}$ of size $d \times 3 d$ to Tx 5. At the same time, Rx 5 feeds back $\mathbf{U}_{5}^{H} \mathbf{H}_{54} \mathbf{v}_{4}^{(1)}$ of size $d \times 3 d$ to Tx 4. Therefore, as illustrated in Fig. 3(b), Tx 5 can avoid causing interference at $\mathrm{Rx} 4$ by using the second auxiliary precoder $\mathbf{v}_{5}^{(2)} \in \mathrm{C}^{3 d \times 2 d}$ which lies in the null space of $\mathbf{U}_{4}^{H} \mathbf{H}_{45} \mathbf{v}_{5}^{(1)}$. Once the first and second auxiliary precoders of Tx 5 are determined, the auxiliary precoder $\mathbf{v}_{5}=\mathbf{v}_{5}^{(1)} \mathbf{v}_{5}^{(2)}$ is obtained at Tx 5. Similarly, Tx 4 can design its second auxiliary precoder $\mathbf{v}_{4}^{(2)} \in \mathrm{C}^{3 d \times 2 d}$ to avoid generating interference at Rx 5, resulting in the acquisition of $\mathbf{v}_{4}=\mathbf{v}_{4}^{(1)} \mathbf{v}_{4}^{(2)}$ at Tx 4 .

3) Phase 3: Tx 4 forwards precoded pilot symbols using its auxiliary precoder $\mathbf{v}_{4}$ which is of size $4 d \times 2 d$. Meanwhile, Tx 5 sends precoded pilot symbols using the auxiliary precoder $\mathbf{v}_{5}$ which is of size $4 d \times 2 d$. As a result, Rxs 1,2 , and 3 are capable of estimating the 
effective channels from Txs 4 and 5.

As shown in Fig. 3(c), by designing the two combining precoders $\tilde{\mathbf{v}}_{4} \in \mathrm{C}^{2 d \times d}$ and $\tilde{\mathbf{v}}_{5} \in \mathrm{C}^{2 d \times d}$, the interferences caused by Tx 4 and Tx 5 can be aligned at each of Rx 2 and $\mathrm{Rx} 3$, i.e.,

$$
\begin{aligned}
& \operatorname{span}\left(\mathbf{u}_{2}^{H} \mathbf{H}_{24} \mathbf{v}_{4} \tilde{\mathbf{v}}_{4}\right)=\operatorname{span}\left(\mathbf{u}_{2}^{H} \mathbf{H}_{25} \mathbf{v}_{5} \tilde{\mathbf{v}}_{5}\right), \\
& \operatorname{span}\left(\mathbf{u}_{3}^{H} \mathbf{H}_{34} \mathbf{v}_{4} \tilde{\mathbf{v}}_{4}\right)=\operatorname{span}\left(\mathbf{u}_{3}^{H} \mathbf{H}_{35} \mathbf{v}_{5} \tilde{\mathbf{v}}_{5}\right) .
\end{aligned}
$$

The combining precoders $\tilde{\mathbf{v}}_{4}$ and $\tilde{\mathbf{v}}_{5}$ can be obtained as

$$
\begin{aligned}
& \tilde{\mathbf{v}}_{4}=\operatorname{eig}\left(\left(\mathbf{u}_{2}^{H} \mathbf{H}_{24} \mathbf{v}_{4}\right)^{-1}\left(\mathbf{u}_{2}^{H} \mathbf{H}_{25} \mathbf{v}_{5}\right)\left(\mathbf{u}_{3}^{H} \mathbf{H}_{35} \mathbf{v}_{5}\right)^{-1}\left(\mathbf{u}_{3}^{H} \mathbf{H}_{34} \mathbf{v}_{4}\right)\right), \\
& \tilde{\mathbf{v}}_{5}=\left(\mathbf{u}_{3}^{H} \mathbf{H}_{35} \mathbf{v}_{5}\right)^{-1}\left(\mathbf{u}_{3}^{H} \mathbf{H}_{34} \mathbf{v}_{4}\right) \tilde{\mathbf{v}}_{4} .
\end{aligned}
$$

To calculate $\tilde{\mathbf{v}}_{4}$ and $\tilde{\mathbf{v}}_{5}$ at $\mathrm{Rx} 3$ according to (14), $\mathrm{Rx} 2$ conveys the matrix $\left(\mathbf{u}_{2}^{H} \mathbf{H}_{24} \mathbf{v}_{4}\right)^{-1}\left(\mathbf{u}_{2}^{H} \mathbf{H}_{25} \mathbf{v}_{5}\right)$ to Rx 3. Once computed, the combining precoders $\tilde{\mathbf{v}}_{4}$ and $\tilde{\mathbf{v}}_{5}$ are delivered from Rx 3 to the corresponding Txs, leading to the acquisition of $\mathbf{V}_{4}=\mathbf{v}_{4} \tilde{\mathbf{v}}_{4}$ and $\mathbf{V}_{5}=\mathbf{v}_{5} \tilde{\mathbf{v}}_{5}$ at Tx 4 and Tx 5, respectively.

Consider the remaining interfering links between Txs 4, 5 and Rxs 1, 2, 3 shown in Fig. 3(c). Since the interference signals from Tx 4 and Tx 5 are shrunk into the $d$ dimensional subspace at each of Rx 2 and $\mathrm{Rx} 3$, each of $\mathrm{Rx} 2$ and $\mathrm{Rx} 3$ with $2 d$ remaining antennas is capable of making its desired signal orthogonal to the subspace spanned by the aligned interferences. Clearly, $\mathrm{Rx} 1$ having $3 d$ remaining antennas can leave $2 d$ dimensions used for the occupation of the interferences from Tx 4 and Tx 5 while maintaining $d$ interference-free dimensions for the desired signal. Eventually, all 20 interfering links can be removed thoroughly through the transceiver design illustrated in Fig. 3.

Table 2. Implementation of the proposed feedback topology for $K=5$

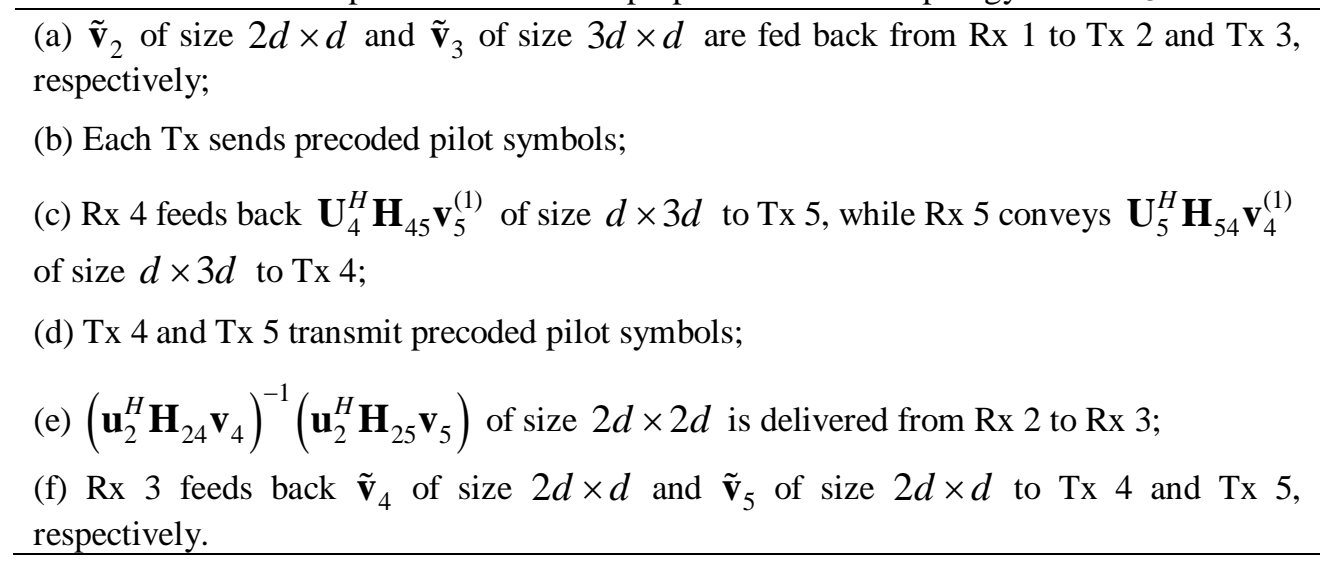

In conclusion, for the case of $K=5$, the proposed feedback design needs 6 time slots to make each IA precoder available at its corresponding Tx, which is shown in Table 2. Adding up the CSI overhead in (a), (c), (e) and (f) in Table 2, the total CSI overhead in the proposed feedback topology for $K=5$ is $19 d^{2}$. 


\subsection{Proposed Feedback Topology for $K \geq 6$}

In this subsection, we extend our feedback design to the case of $K \geq 6$. For the $K \geq 6$ case where each node is equipped with $(K-1) d$ antennas, dimensionality-decreasing is applied at each Tx. To be specific, we randomly generate a precoder of size $(K-1) d \times d$ at each of Txs $1,2, \ldots,(K-4)$, independently, so that each of these Txs equivalently has $d$ remaining antennas after applying the corresponding precoder. Additionally, we randomly pick the auxiliary precoders $\mathbf{v}_{K-3} \in \mathrm{C}^{(K-1) d \times 2 d}, \mathbf{v}_{K-2} \in \mathrm{C}^{(K-1) d \times(K-2) d}$ for $\mathrm{Tx}(K-3)$, Tx $(K-2)$ respectively, as well as the first auxiliary precoders $\mathbf{v}_{K-1}^{(1)} \in \mathrm{C}^{(K-1) d \times(K-2) d}$, $\mathbf{v}_{K}^{(1)} \in \mathrm{C}^{(K-1) d \times(K-2) d}$ for $\mathrm{Tx}(K-1)$, Tx $K$ respectively. After applying these auxiliary precoders (or first auxiliary precoders), Tx $(K-3)$ equivalently has $2 d$ remaining antennas, and each of Txs $(K-2),(K-1), K$ equivalently remains $(K-2) d$ antennas.

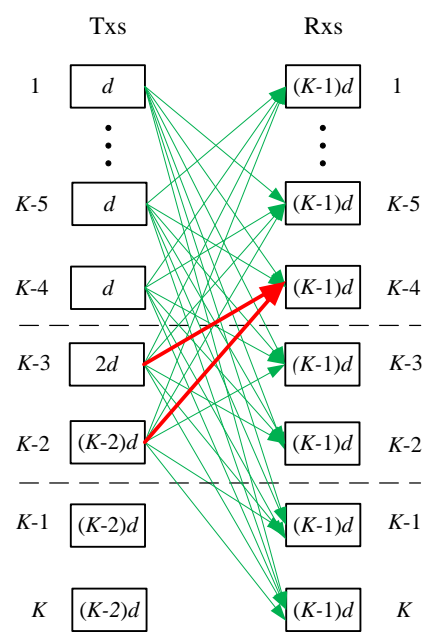

(a) Phase 1

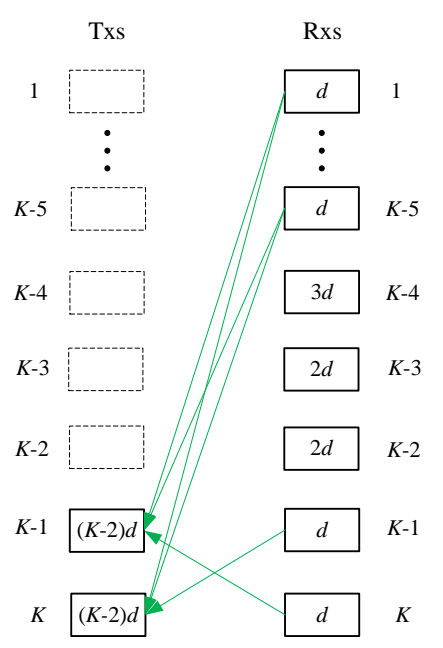

(b) Phase 2

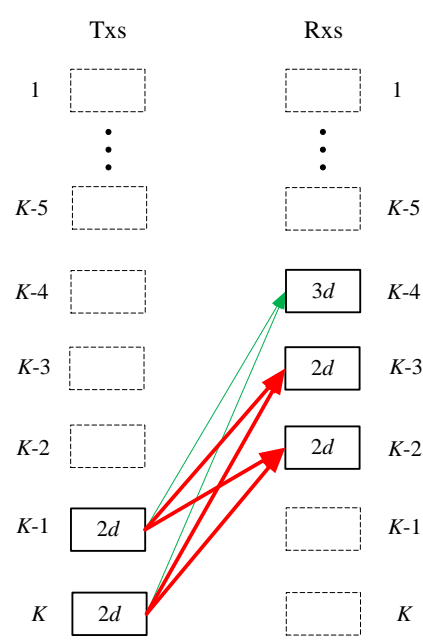

(c) Phase 3

Fig. 5. Illustration of how to eliminate all cross-link interferences when $K \geq 6$

1) Phase 1: As shown in Fig. 5(a), due to the remaining antennas at Txs $(K-3)$ and $(K-2)$, the interference signals from these two Txs to $\mathrm{Rx}(K-4)$ can be aligned in the same subspace at $\mathrm{Rx}(K-4)$ by designing the combining precoders $\tilde{\mathbf{v}}_{K-3} \in \mathrm{C}^{2 d \times d}$ and $\tilde{\mathbf{v}}_{K-2} \in \mathrm{C}^{(K-2) d \times d}$. Once determined, $\tilde{\mathbf{v}}_{K-3}$ and $\tilde{\mathbf{v}}_{K-2}$ are conveyed from $\mathrm{Rx}(K-4)$ to $\mathrm{Tx}$ $(K-3)$ and $\mathrm{Tx}(K-2)$ for the construction of the precoders $\mathbf{V}_{K-3}$ and $\mathbf{V}_{K-2}$ respectively. Then each of Txs $1,2, \ldots,(K-2)$ sends precoded pilot symbols using its precoder of size $(K-1) d \times d$, while Tx $(K-1)$ and Tx $K$ forward precoded pilot symbols using $\mathbf{v}_{K-1}^{(1)}$ and $\mathbf{v}_{K}^{(1)}$ respectively, for the purpose of channel estimation at the Rx side. 


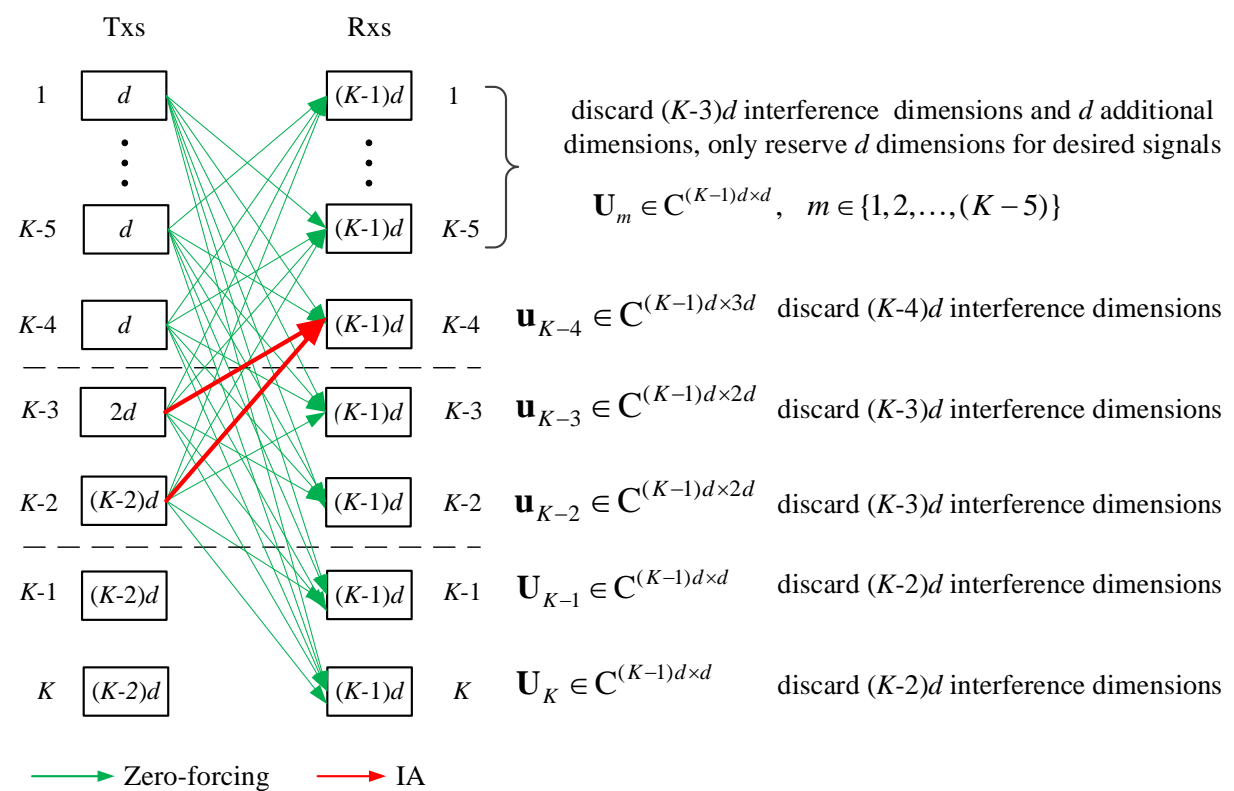

Fig. 6. Illustration of the elimination of interferences from Txs $1,2, \ldots,(K-2)$ at the Rx side during phase 1 when $K \geq 6$

After channel estimation, each Rx removes the interferences caused by Txs $1,2, \ldots$, $(K-2)$, leading to the determination of the receive filters (or auxiliary receive filters) as shown in Fig. 6.

Table 3. Implementation of the proposed feedback topology for $K \geq 6$

(a) Rx $(K-4)$ feeds back $\tilde{\mathbf{v}}_{K-3} \in \mathrm{C}^{2 d \times d}$ and $\tilde{\mathbf{v}}_{K-2} \in \mathrm{C}^{(K-2) d \times d}$ to Tx $(K-3)$ and Tx $(K-2)$, respectively;

(b) Each Tx sends precoded pilot symbols;

(c) $\mathrm{Rx} m, m \in\{1,2, \ldots,(K-5)\}$, feeds back the $d \times(K-2) d$ matrices $\mathbf{U}_{m}^{H} \mathbf{H}_{m(K-1)} \mathbf{v}_{K-1}^{(1)}$ and $\mathbf{U}_{m}^{H} \mathbf{H}_{m K} \mathbf{v}_{K}^{(1)}$ to Tx $(K-1)$ and Tx $K$ respectively, Rx $K$ feeds back the $d \times(K-2) d$ matrix $\mathbf{U}_{K}^{H} \mathbf{H}_{K(K-1)} \mathbf{v}_{K-1}^{(1)}$ to $\mathrm{Tx}(K-1)$, and $\operatorname{Rx}(K-1)$ conveys $\mathbf{U}_{K-1}^{H} \mathbf{H}_{(K-1) K} \mathbf{v}_{K}^{(1)}$ of size $d \times(K-2) d$ to Tx $K$;

(d) Tx $(K-1)$ and Tx $K$ transmit precoded pilot symbols;

(e) Rx $(K-3)$ delivers the matrix $\left(\mathbf{u}_{K-3}^{H} \mathbf{H}_{(K-3)(K-1)} \mathbf{v}_{K-1}\right)^{-1}\left(\mathbf{u}_{K-3}^{H} \mathbf{H}_{(K-3) K} \mathbf{v}_{K}\right)$ of size $2 d \times 2 d$ to $\mathrm{Rx}(K-2)$;

(f) $\operatorname{Rx}(K-2)$ feeds back $\tilde{\mathbf{v}}_{K-1} \in \mathrm{C}^{2 d \times d}$ and $\tilde{\mathbf{v}}_{K} \in \mathrm{C}^{2 d \times d}$ to $\mathrm{Tx}(K-1)$ and $\mathrm{Tx} K$, respectively.

2) Phase 2: In this phase, as illustrated in Fig. 5(b), the second auxiliary precoder $\mathbf{v}_{K-1}^{(2)} \in \mathrm{C}^{(K-2) d \times 2 d}$ is designed to avoid creating interference at $\mathrm{Rx} m, m \in\{1,2, \ldots,(K-5)\}$, as well as $\mathrm{Rx} K$. Similarly, $\mathbf{v}_{K}^{(2)} \in \mathrm{C}^{(K-2) d \times 2 d}$ is determined by avoiding causing interference at $\mathrm{Rx} m$ and $\operatorname{Rx}(K-1)$. Therefore, in order to calculate $\mathbf{v}_{K-1}^{(2)}$ and $\mathbf{v}_{K}^{(2)}$ at $\mathrm{Tx}(K-1)$ and 
Tx $K$ respectively, we implement step (c) of the proposed feedback topology shown in Table 3.

3) Phase 3: Once Tx $(K-1)$ and $\mathrm{Tx} K$ acquire the auxiliary precoders $\mathbf{v}_{K-1}=\mathbf{v}_{K-1}^{(1)} \mathbf{v}_{K-1}^{(2)}$ and $\mathbf{v}_{K}=\mathbf{v}_{K}^{(1)} \mathbf{v}_{K}^{(2)}$ respectively, these two Txs send precoded pilot symbols using the corresponding auxiliary precoders. Following the similar manner as that introduced in phase 3 for the case of $K=5$, step (e) of the proposed feedback topology in Table 3 is carried out to compute the combining precoders $\tilde{\mathbf{v}}_{K-1}$ and $\tilde{\mathbf{v}}_{K}$. Then the two computed combining precoders are fed back from $\mathrm{Rx}(K-2)$ to the corresponding Txs.

Finally, all interferences in the system can be eliminated completely. Adding up the CSI overhead in (a), (c), (e) and (f) in Table 3, the total CSI overhead in the proposed feedback topology for $K \geq 6$ is $\left(2 K^{2}-11 K+24\right) d^{2}$.

Remark 1 (Tx Selection Criteria): When performing dimensionality-decreasing at the Tx side, the Tx selection order is not unique. For example, in the case of $K=5$, after implementing dimensionality-decreasing, we let Txs $1,2,3,4,5$ equivalently remain $d, 2 d$, $3 d, 3 d, 3 d$ antennas respectively in the proposed feedback topology, or alternatively we can set Txs 2, 1, 3, 4, 5 to equivalently have $d, 2 d, 3 d, 3 d, 3 d$ remaining antennas respectively. Although the Tx selection order has a direct impact on the choice of Txs and Rxs that participate in the alignment process, it does not affect the achievable DoF and the CSI overhead. For simplicity of exposition, in the proposed feedback topology, the Tx selection for dimensionality-decreasing is carried out in ascending order of the number of remaining antennas.

Remark 2 (Antenna Selection Criteria): A precoding method is used for implementing dimensionality-decreasing to allow a certain Tx to remain $N$ (where $d \leq N<M$ ) antennas in the proposed feedback topology. Also, the random antenna selection can be employed to achieve this goal by randomly selecting $N$ antennas and powering off others. If we exploit other antenna selection criterions, such as maximum post-processing SNR, maximum capacity [25], additional feedback information is required because a Rx delivers the optimal subset of transmit antennas to the corresponding Tx [25]. Note that the antenna selection and Tx selection mentioned in Remark 1 can be jointly optimized to improve the diversity gain at the cost of increased feedback overhead. Since the CSI overhead is the focus of this work, we leave a joint optimization of antenna selection and Tx selection with no impact on multiplexing gain (i.e., DoF) as the future work.

Remark 3 (Forward Channel Training): In the proposed feedback topology, it is assumed that multiple Txs can simultaneously transmit orthogonal pilots with which the RXs are able to accurately estimate the cross-link forward channels. Note that this assumption is also exploited in the four-hop feedback topology in [13]. Although all existing feedback topologies except the four-hop topology require the acquisition of two estimated forward channels at each Rx to calculate IA precoders, the initial forward channel training procedure (i.e., initial pilot sending) of these five topologies is omitted in [12] and [13]. For the proposed feedback topology, it requires initial pilot sending, and also needs pilot sending in time slot 2 and time slot 4. It is clear that pilot transmission incurs pilot overhead [26]. Ignoring the pilot overhead caused by the initial pilot sending, the transmission of training sequences in time slots 2 and 4 in the proposed feedback topology results in additional pilot overhead. Due to the fact that the CSI problem is a challenging issue for the application of IA [5], [14], we mainly concentrate on the CSI overhead in this work. 


\section{Comparison with Existing Topologies}

For the proposed feedback topology, at the Tx side, each column of a precoder is normalized to have unit norm after this precoder is obtained at its target Tx. Then equal power allocation is applied as in [12]. On the other hand, at the Rx side, once a receive filter is determined, it is normalized to unit norm at each column as in [12]. By employing the above design, from equation (2), the proposed feedback topology has the same performance as those feedback topologies in [12] in terms of average sum rate. Notably, [13] has not provided the power allocation strategy and the process of normalizing transmit/receive filters. Actually, if waterfilling power allocation, which is optimal in terms of sum rate, is employed, each Tx should know how to allocate power to data streams, resulting in extra feedback overhead. Therefore, equal power allocation requiring no feedback information about how much power should be allocated to each data stream is a feedback-saving strategy, and thus it is exploited in this paper and [12]. In summary, under the condition of equal power allocation and normalized transmit/receive filters, the feedback topologies in [12], [13] and this paper yield the same average sum rate. From the above discussions, as in [12] for the perfect CSI case and [13], in this section we only present the comparison of CSI overhead among various feedback topologies with the same DoF.

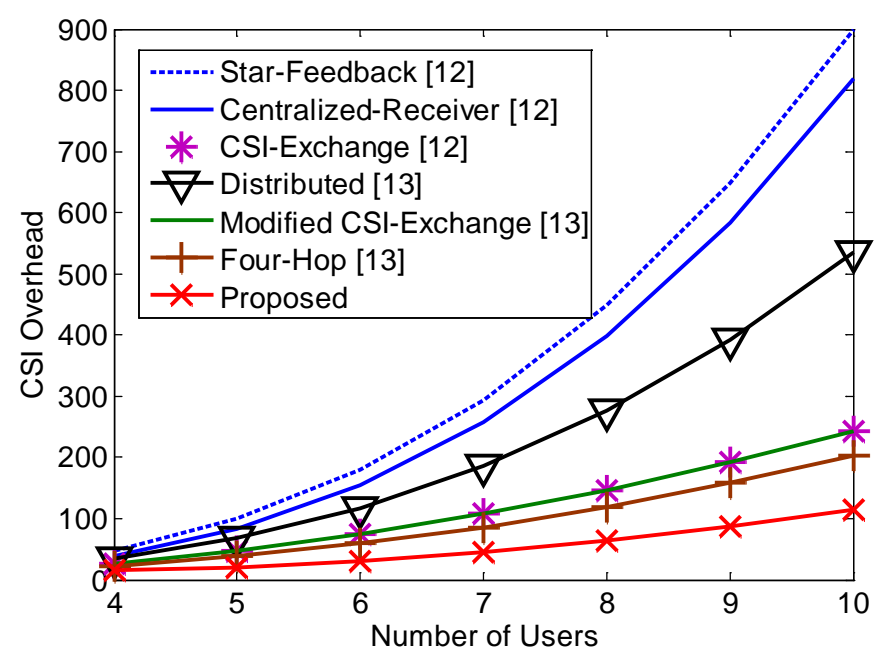

Fig. 7. CSI overhead comparison for the proposed feedback topology and existing topologies for the case of $d=1$

In Fig. 7, we provide the CSI overhead comparison for the existing feedback topologies in [12] and [13] and our proposed design according to different numbers of users. As can be seen in this figure, the proposed feedback topology yields a significant saving in CSI feedback overhead compared to the existing six feedback structures, meaning that a further saving of CSI overhead can be achieved in the field of feedback topology construction in spite of the existence of previously designed topologies. The saving of CSI overhead obtained by the proposed design comes from an efficient exploitation of dimensionality-decreasing and selection of a subset of Rxs receiving aligned interferences. Additionally, considering that the CSI-exchange topology and the four-hop design are the feedback topologies with the lowest CSI overheads in [12] and [13] respectively, a more detailed comparison of the feedback cost for these two feedback strategies and our proposed structure is illustrated in Table 4. This table further highlights the advantage of the proposed feedback topology in terms of CSI 
overhead reduction. For example, for the case of $K=5$, the CSI overhead of the proposed design is half that of the four-hop structure.

In addition, consider the required number of time slots for the acquisition of IA precoders at the Tx side, which has a direct impact on the robustness of the transmission against channel variations. The required number of time slots for the proposed feedback topology is 6 , which is equal to (for $K=4$ ) or less than (for $K \geq 5$ ) that of the CSI-exchange topology operating over $2(K-1)$ time slots, but greater than that of the four-hop structure operating over 4 time slots. Hence, it would be interesting in our future work to investigate the tradeoff between the CSI overhead reduction and the time slots needed for precoder acquisition.

Table 4. CSI overhead comparison according to number of users $K$

\begin{tabular}{|c|c|c|c|c|c|c|c|}
\hline \multirow{2}{*}{$\begin{array}{c}\text { Feedback } \\
\text { Topologies }\end{array}$} & \multicolumn{7}{|c|}{ CSI Overhead $(\boldsymbol{d}=\mathbf{1})$} \\
\cline { 2 - 8 } & $\boldsymbol{K = 4}$ & $\boldsymbol{K}=\mathbf{5}$ & $\boldsymbol{K}=\mathbf{6}$ & $\boldsymbol{K}=\mathbf{7}$ & $\boldsymbol{K}=\mathbf{8}$ & $\boldsymbol{K}=\mathbf{9}$ & $\boldsymbol{K}=\mathbf{1 0}$ \\
\hline \hline CSI-Exchange [12] & 27 & 48 & 75 & 108 & 147 & 192 & 243 \\
\hline Four-Hop [13] & 23 & 38 & 59 & 86 & 119 & 158 & 203 \\
\hline Proposed & 15 & 19 & 30 & 45 & 64 & 87 & 114 \\
\hline
\end{tabular}

\section{Conclusion}

By arranging the feedback links in interference networks, feedback topology can reduce the CSI feedback overhead to an acceptable level. Although several feedback topologies have been presented in previous works, the design of feedback-saving strategy is still a research direction for facilitating the practical utilization of IA. In this paper, to seek a further reduction of CSI overhead, we propose a new feedback topology. The key idea of the proposed feedback topology is an efficient utilization of dimensionality-decreasing at the Tx side combined with the selection of a subset of Rxs receiving aligned interference signals. We illustrate the superiority of the proposed feedback design with respect to CSI overhead compared to the existing feedback topologies.

\section{References}

[1] H. Chae, K. Kim, R. Ran and D. K. Kim, "A single feedback based interference alignment for three-user MIMO interference channels with limited feedback," KSII Trans. on Internet and Information systems, vol. 7, no. 4, pp. 692-710, Apr. 2013. Article (CrossRef Link)

[2] Q. Wang, Y. Shu, M. Dong, J. Xu and X. Tao, "Degrees of freedom of 3-user MIMO interference channels with instantaneous relay using interference alignment," KSII Trans. on Internet and Information systems, vol. 9, no. 5, pp. 1624-1641, May 2015. Article (CrossRef Link)

[3] X. Jiang and B. Zheng, "Interference alignment in two-way relay channel with compute-and-forward,” KSII Trans. on Internet and Information systems, vol. 10, no. 2, pp. 593-607, Feb. 2016. Article (CrossRef Link)

[4] Q. Niu, Z. Zeng, T. Zhang and Z. Hu, "Interference alignment based transceiver design in OSG mode of HetNets," KSII Trans. on Internet and Information systems, vol. 9, no. 6, pp. 2014-2034, Jun. 2015. Article (CrossRef Link)

[5] O. El Ayach, S.W. Peters, and R. W. Heath, Jr., "The practical challenges of interference alignment,” IEEE Wireless Commun., vol. 20, no. 1, pp. 35-42, Feb. 2013. Article (CrossRef Link)

[6] M. Rezaee, M. Guillaud, and F. Lindqvist, "CSIT sharing over finite capacity backhaul for spatial interference alignment,” in Proc. of IEEE Int. Symp. Inform. Theory, pp. 569-573, Jul. 2013. Article (CrossRef Link) 
[7] M. Rezaee and M. Guillaud, "CSIT sharing for spatial interference alignment using limited capacity backhaul,” IEEE Trans. Wireless Commun., vol. 14, no. 12, pp. 6843-6853, Dec. 2015. Article (CrossRef Link)

[8] J. Shi, Q. Luo, and M. You, "An efficient method for enhancing TDD over the air reciprocity calibration," in Proc. of IEEE Wireless Commun. and Networking Conf., pp. 339-344, Mar. 2011. Article (CrossRef Link)

[9] S. Han, C. Yang, G. Wang, D. Zhu, and M. Lei, "Coordinated multipoint transmission strategies for TDD systems with non-ideal channel reciprocity,” IEEE Trans. Commun., vol. 61, no. 10, pp. 4256-4270, Oct. 2013. Article (CrossRef Link)

[10] J. Thukral and H. Bolcskei, "Interference alignment with limited feedback," in Proc. of IEEE Int. Symp. Inform. Theory, pp. 1759-1763, Jun. 2009. Article (CrossRef Link)

[11] R. T. Krishnamachari and M. K. Varanasi, "Interference alignment under limited feedback for MIMO interference channels,” in Proc. of IEEE Int. Symp. Inform. Theory, pp. 619-623, Jun. 2010. Article (CrossRef Link)

[12] S. Cho, H. Chae, K. Huang, D. Kim, V. Lau, H. Seo, and B. Kim, "Feedback-topology designs for interference alignment in MIMO interference channels," IEEE Trans. Signal Process., vol. 60, no. 12, pp. 6561-6575, Dec. 2012. Article (CrossRef Link)

[13] J. Jin, L. Li, H. Tian, Q. Wang, and X. Gao, "New feedback topology designs with reduced CSI overhead for MIMO interference alignment," IEEE Signal Process. Lett., vol. 21, no. 2, pp. 176-179, Feb. 2014. Article (CrossRef Link)

[14] N. Zhao, F. R. Yu, M. Jin, Q. Yan, and V. C. M. Leung, "Interference alignment and its applications: A survey, research issues and challenges,” IEEE Commun. Surveys Tuts., vol. 18, no. 3, pp. 1779-1803, Mar. 2016. Article (CrossRef Link)

[15] G. Bresler, D. Cartwright, and D. Tse, "Feasibility of interference alignment for the MIMO interference channel,” IEEE Trans. Inf. Theory, vol. 60, no. 9, pp. 5573-5586, Sep. 2014. Article (CrossRef Link)

[16] O. Gonzalez, C. Beltran, and I. Santamaria, "On the number of interference alignment solutions for the K-User MIMO channel with constant coefficients,” IEEE Trans. Inf. Theory, vol. 61, no. 11, pp. 6028-6048, Nov. 2015. Article (CrossRef Link)

[17] X. Rao, L. Ruan, and V. K. N. Lau, "CSI feedback reduction for MIMO interference alignment," IEEE Trans. Signal Process., vol. 61, no. 18, pp. 4428-4437, Sep. 2013. Article (CrossRef Link)

[18] P. de Kerret and D. Gesbert, "Interference alignment with incomplete CSIT sharing," IEEE Trans. Wireless Commun., vol. 13, no. 5, pp. 2563-2573, May 2014. Article (CrossRef Link)

[19] O. El Ayach and R. W. Heath, Jr., "Grassmannian differential limited feedback for interference alignment,” IEEE Trans. Signal Process., vol. 60, no. 12, pp. 6481-6494, Dec. 2012. Article (CrossRef Link)

[20] L. Zhang, L. Song, M. Ma, Z. Zhang, M. Lei, and B. Jiao, "Interference alignment with differential feedback,” IEEE Trans. Veh. Technol., vol. 61, no. 6, pp. 2878-2883, Jul. 2012. Article (CrossRef Link)

[21] O. El Ayach and R. W. Heath, Jr., "Interference alignment with analog channel state feedback," IEEE Trans. Wireless Commun., vol. 11, no. 2, pp. 626-636, Feb. 2012. Article (CrossRef Link)

[22] O. El Ayach, A. Lozano, and R. W. Heath Jr., "On the overhead of interference alignment: Training, feedback, and cooperation,” IEEE Trans. Wireless Commun., vol. 11, no. 11, pp. 4192-4203, Nov. 2012. Article (CrossRef Link)

[23] X. Rao and V. K. N. Lau, "Interference alignment with partial CSI feedback in MIMO cellular networks,” IEEE Trans. Signal Process., vol. 62, no. 8, pp. 2100-2110, Apr. 2014. Article (CrossRef Link)

[24] X. Rao and V. K. N. Lau, "Minimization of CSI feedback dimension for interference alignment in MIMO interference multicast networks," IEEE Trans. Inf. Theory, vol. 61, no. 3, pp. 1218-1246, Mar. 2015. Article (CrossRef Link)

[25] R. W. Heath, Jr., S. Sandhu, and A. Paulraj, "Antenna selection for spatial multiplexing systems with linear receivers,” IEEE Commun. Lett., vol. 5, no. 4, pp. 142-144, Apr. 2001. Article (CrossRef Link) 
[26] R. K. Mungara, G. George, and A. Lozano, "Overhead and spectral efficiency of pilot-assisted interference alignment in time-selective fading channels,” IEEE Trans. Wireless Commun., vol. 13, no. 9, pp. 4884-4895, Sep. 2014. Article (CrossRef Link)

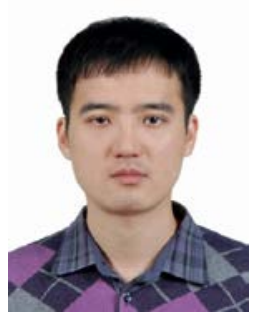

Jin Jin received the Ph.D. degree in communications and information systems from Beijing University of Posts and Telecommunications, Beijing, China, in 2014. Since 2014, he has been with the School of Information Engineering, Zhengzhou University, where he is currently a Lecturer. His research interests include cooperative communications and interference alignment.

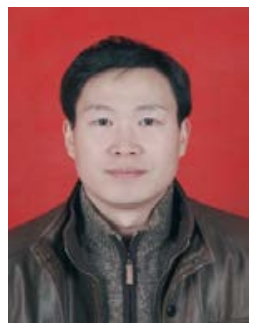

Xiang-Chuan Gao received the B.Sc. and M.Eng. degrees from Zhengzhou University, Zhengzhou, China, in 2005 and 2008, respectively, and the Ph.D. degree from Beijing University of Posts and Telecommunications, Beijing, China, in 2011. He is currently an Associate Professor with the School of Information Engineering, Zhengzhou University. His research interests include massive MIMO, cooperative communications, and visible light communication.

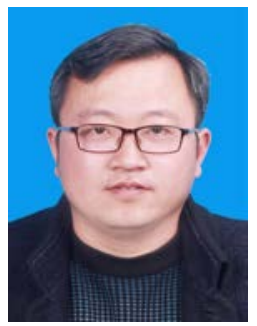

Xingwang Li received his B.Sc. degree in communication engineering from Henan Polytechnic University, China, in 2007. He then received the M.Sc. degree from the National Key Laboratory of Science and Technology on Communications at University of Electronic Science and Technology of China (UESTC) and Ph.D. degree in communication and information system from the State Key Laboratory of Networking and Switching Technology at Beijing University of Posts and Telecommunications (BUPT). He is currently a lecturer with the School of Physics and Electronic Information Engineering, Henan Polytechnic University, Jiaozuo China. He has several papers published in journal and conferences, has authored several patents and he has worked on several funded research projects on the wireless communications areas. His research interests include MIMO communication, cooperative communication, hardware constrained communication, non-orthogonal multiple access (NOMA), physical layer security, FSO communications, and performance analysis of fading channels. 


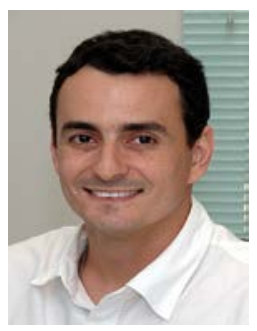

Charles Casimiro Cavalcante received the B.Sc and M.Sc in Electrical Engineering from the Federal University of Cear (UFC), Brazil, in 1999 and 2001, respectively, and the Ph.D. degree from the University of Campinas (UNICAMP), Brazil, in 2004. He has held a grant for Scientific and Technological Development from 2004 to 2007 and since March 2009 he has a grant of Scientific Research Productivity both from the Brazilian Research Council (CNPq). From March 2007 to November 2008 he was a Visiting Professor at Teleinformatics Engineering Department of UFC and since November 2008 he is an Assistant Professor at the same department and university holding the Statistical Signal Processing chair. From August 2014 to July 2015 he was a Visiting Assistant Professor at the Department of Computer Science and Electrical Engineering (CSEE) from University of Maryland, Baltimore County (UMBC) in the United States. He has been working on signal processing strategies for communications where he has several papers published in journal and conferences, has authored three international patents and he has worked on several funded research projects on the signal processing and wireless communications areas. He is also a co-author of the book Unsupervised Signal Processing: Channel Equalization and Source Separation, published by CRC Press. He is a researcher of the Wireless Telecommunications Research Group (GTEL) where he leads research on signal processing and wireless communications. Dr. Cavalcante is a Senior Member of the IEEE and Senior Member of the Brazilian Telecommunications Society (SBrT). His main research interests are in signal processing for communications, blind source separation, wireless communications, and statistical signal processing.

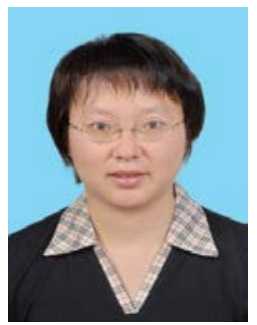

Lihua Li received her doctor degree in 2004 at Beijing University of Posts and Telecommunications (BUPT). She is currently an associate professor in BUPT. She had been a short -term visiting scholar at Brunel University in UK in 2006. And she visited the University of Oulu from August 2010 to August 2011. Her research focuses on wideband mobile communication technologies including MIMO, link adaptation, cooperative transmission technologies etc. relating to new generation mobile communication systems such as LTE and IMT-Advanced. She has published 63 papers in international and domestic journals and academic conferences, and 5 books. She has applied 20 national invention patents and one international patent. She was selected and funded as one of the New Century Excellent Talents by the Chinese Ministry of Education in 2008. She has won the second prize of China State Technological Invention Award (the 1st level award in China) in 2008 and the first prize of China Institute of Communications Science and Technology Award in 2006 for her research achievements of "Wideband Wireless Mobile TDD-OFDM -MIMO Technologies”. She has served as a group leader in 3GPP LTE RAN1 standardization work on behalf of BUPT in 2005, when she submitted more than 20 relevant proposals to 3GPP LTE and 7 of them were accepted. She has taken part in China IMT-Advanced technology work group since 2007. And so far she has submitted 33 relevant proposals, 15 of which were accepted. 